\title{
Analytical functions for the calculation of hyperspherical potential curves of atomic systems
}

\author{
J. J. De Groote, ${ }^{1}$ Mauro Masili, ${ }^{2}$ and J. E. Hornos ${ }^{3}$ \\ ${ }^{1}$ Instituto de Química de Araraquara, Universidade Estadual Paulista, Caixa Postal 355, 14 801-970 Araraquara, SP, Brazil \\ ${ }^{2}$ Department of Physics and Astronomy, The University of Nebraska, 116 Brace Laboratory, Lincoln, Nebraska $68588-0111$ \\ ${ }^{3}$ Instituto de Física de São Carlos, Universidade de São Paulo, Caixa Postal 369, 13 560-970 São Carlos, SP, Brazil
}

(Received 8 March 2000; revised manuscript received 25 April 2000; published 15 August 2000)

\begin{abstract}
We present angular basis functions for the Schrödinger equation of two-electron systems in hyperspherical coordinates. By using the hyperspherical adiabatic approach, the wave functions of two-electron systems are expanded in analytical functions, which generalizes the Jacobi polynomials. We show that these functions, obtained by selecting the diagonal terms of the angular equation, allow efficient diagonalization of the Hamiltonian for all values of the hyperspherical radius. The method is applied to the determination of the ${ }^{1} S^{e}$ energy levels of the $\mathrm{Li}^{+}$and we show that the precision can be improved in a systematic and controllable way.
\end{abstract}

PACS number(s): 31.25.-v, 31.15.Ja, 31.15.Ar

\section{INTRODUCTION}

Hyperspherical coordinates have been used, for a long time, to solve the $N$-body quantum problems in molecular [1-5], atomic [6-11], and nuclear physics [12-14]. The standard approach begins with the introduction of Jacobi variables to eliminate the center-of-mass coordinates. The remaining $3 N-3$ degrees of freedom are described by a (hyper-) radius $R$ and $3 N-4$ angular variables $\Omega$ $=\left(\omega_{1}, \ldots, \omega_{3 N-4}\right)$ composed by $N-2$ hyperspherical angular variables and by the $2 N-2$ usual spherical angular variables $\theta_{i}$ and $\phi_{i}$. The nonrelativistic kinetic-energy operator is separable on those variables and its angular part can be identified with the Casimir operator of the $\mathrm{O}(3 N-3)$ symmetry group. The problem of constructing angular basis functions, which diagonalize the kinetic operator, is reduced to the well-established problem of construction of harmonic functions for the orthogonal groups. The irreducible representations of these groups can be labeled by a set $\lambda$ of integer indices, and angular functions can be constructed using Jacobi polynomials for each representation.

In concrete applications, the attention is focused on the properties of the wave functions; however, the procedure is a part of the so-called algebraic methods [13], in which a symmetry group $G$ is decomposed in a chain of subgroups $G \supset G_{1} \supset \cdots \supset \mathrm{O}(3)$ ending in the tridimensional rotation group. The multidimensional orthogonal symmetry is broken, not spontaneously by its subgroups but by the interparticle interactions. The effects on the calculation will be the lack of convergence in the expansion by harmonic functions and the need of a large basis to reproduce the wave functions. The intensity of this effect will depend on how "badly" the symmetry was broken. The Coulombic threebody systems are an illustrative example on this process for which we usually choose the $\mathrm{O}(6) \supset \mathrm{O}(3) \times \mathrm{O}(3) \supset \mathrm{O}(3)$ chain. The $\mathrm{O}(3) \times \mathrm{O}(3)$ symmetry is lightly broken, leading to a reasonable convergence in the composed angular momentum function, which characterizes this subgroup. In opposition, the $\mathrm{O}(6)$ symmetry is strongly broken due to the long range of the electromagnetic interaction. A much more favorable situation occurs in the nuclear problem, for which the short-range forces confine the particles, enhancing the role of the kinetic terms. As a result, calculations of the wave function for nuclei with a mass number between two and ten have been reported [12]. In the atomic counterpart, the applications are mainly limited to the three- and four-body case.

An alternative approach to the use of the multidimensional orthogonal basis is to solve the partial differential equation for the angular variables directly by expanding the angular function in a power series in appropriated variables [7]. The functions will not be eigenstates of the orthogonal group but will fully incorporate the interparticle interactions. This approach has been used in calculations of potential curves for helium, $\mathrm{H}^{-}, \mathrm{D}_{2}^{+}$, $\mathrm{DD} \mu$, excitons and other species $[2,15,16]$. The inclusion of nonadiabatic couplings has been reported and accurate ground-state [17] and excitedstate [18] energies were obtained.

The generalization of the hyperspherical adiabatic approach (HAA) to complex atoms will require the solution of an infinite set of partial equations instead of ordinary equations as in the three-body case, which is unpractical even in the lithium case. With this in mind, we reviewed the HAA, searching for a basis that can be used as building blocks for atomic and molecular calculations. The set of functions depends parametrically on the hyperspherical radius $R$. At the $R=0$ limit, they reproduce the Jacobi polynomials and at the $R \rightarrow \infty$ limit, the Laguerre functions behavior, which characterizes the Coulombic problem, is exactly achieved. The functions are obtained extracting the diagonal part of the interactions for all angular momentum manifolds. The functions are transcendental but their Taylor expansion coefficients can be calculated with arbitrary precision.

In order to verify the efficiency of our procedure, we analyze the potential curves and nonadiabatic couplings for $\mathrm{Li}^{+}$ and compare the corresponding lowest energies with values obtained by other methods. We observed that the curves have been calculated accurately and the long-range problems are absent.

This paper is organized as follows. In Sec. II, we review the HAA approach in order to establish the problem. In Sec. III, we construct what we refer to as "Laguerre-Jacobi" functions and we discuss their properties. In Sec. IV, we 
present the solutions of the method for the $\mathrm{Li}^{+}$ion and finally, Sec. V is dedicated to the conclusion.

\section{HYPERSPHERICAL ADIABATIC APPROACH}

The HAA is an adequate method to treat $N$-body systems interacting with the long-range Coulombian forces due to its molecularlike description that brings to mind the spirit of the Born-Oppenheimer approximation. With the choice of appropriate Jacobi coordinates, the center-of-mass degrees of freedom can be excluded and the hyperspherical coordinates are built in order to correlate the remaining $N-1$ radial coordinates. Those coordinates are composed to bring about only one radial component $R$,

$$
R^{2}=\sum_{i=1}^{N-1} r_{i}^{2} \quad(0 \leqslant R \leqslant \infty)
$$

and also angular variables that can be related to the Jacobi radial coordinates $\vec{r}_{1}, \vec{r}_{2}, \ldots, \vec{r}_{N-1}$ as [12]

$$
\begin{gathered}
r_{1}=R \sin \left(\alpha_{N-2}\right) \cdots \sin \left(\alpha_{2}\right) \sin \left(\alpha_{1}\right), \\
r_{2}=R \sin \left(\alpha_{N-2}\right) \cdots \sin \left(\alpha_{2}\right) \cos \left(\alpha_{1}\right), \\
r_{3}=R \sin \left(\alpha_{N-2}\right) \cdots \cos \left(\alpha_{2}\right), \\
\vdots \\
r_{N-2}=R \sin \left(\alpha_{N-2}\right) \cos \left(\alpha_{N-3}\right), \\
r_{N-1}=R \cos \left(\alpha_{N-2}\right) \quad\left(0 \leqslant \alpha_{i} \leqslant \pi / 2\right) .
\end{gathered}
$$

With those coordinates, the hyperspherical Schrödinger equation has the compact form

$$
\left[\frac{d^{2}}{d R^{2}}+\frac{(3 N-4)}{R} \frac{d}{d R}+\frac{\hat{U}(R ; \Omega)}{R^{2}}+2 \varepsilon\right] \psi(R, \Omega)=0
$$

where $\varepsilon$ is the system energy and the operator $\hat{U}(R ; \Omega)$ depends on all compact variables $\Omega=\left(\alpha_{i}, \phi_{j}, \theta_{j} ; i=1, \ldots, N\right.$ $-2 ; j=1, \ldots, N-1)$ and on the hyperradius $R$ through the expression

$$
\hat{U}=C_{2}[O(3 N-3)]+R \hat{V}(R ; \Omega),
$$

where $C_{2}$ is the Casimir operator of the $\mathrm{O}(3 N-3)$ group and $\hat{V} / R$ is the interparticle potential energy. In the case of Coulombic interaction, $\hat{V}$ is independent of $R$. This means a simple linear dependence on $R$ that the HAA exploits fully using this coordinate as an adiabatic one. Similarly to the Born-Oppenheimer method, one angular equation is defined:

$$
\hat{U}(R ; \Omega) \Phi_{\lambda}(R ; \Omega)=U_{\lambda}(R) \Phi_{\lambda}(R ; \Omega),
$$

for each parametrized value of $R$. The eigenvalues are usually called potential curves and the corresponding eigenfunctions are the channel functions constructed for each $\mathrm{O}(3 \mathrm{~N}$
$-3)$ representation. The set $\lambda$ represents the quantum numbers that label the channel functions.

Finally, the wave function is expanded in the channel functions

$$
\psi(R, \Omega)=R^{-(3 N-4) / 2} \sum_{\lambda} F_{\lambda}(R) \Phi_{\lambda}(R ; \Omega)
$$

resulting in an infinite coupled set of ordinary differential equations for the radial amplitude

$$
\begin{aligned}
& {\left[\frac{d^{2}}{d R^{2}}+\frac{3(3 N-4)(2-N)}{4 R}+\frac{U_{\lambda}(R)}{R^{2}}+2 \varepsilon\right] F_{\lambda}(R)} \\
& +\sum_{\lambda^{\prime}} W_{\lambda \lambda^{\prime}}(R) F_{\lambda^{\prime}}(R)=0
\end{aligned}
$$

where

$$
W_{\lambda \lambda^{\prime}}(R)=2 P_{\lambda \lambda^{\prime}}(R) \frac{d}{d R}+Q_{\lambda \lambda^{\prime}}(R)
$$

are the nonadiabatic coupling terms with

$$
\begin{gathered}
P_{\lambda \lambda^{\prime}}(R)=\left\langle\Phi_{\lambda}\left|\frac{d}{d R}\right| \Phi_{\lambda^{\prime}}\right\rangle, \\
Q_{\lambda \lambda^{\prime}}(R)=\left\langle\Phi_{\lambda}\left|\frac{d^{2}}{d R^{2}}\right| \Phi_{\lambda^{\prime}}\right\rangle .
\end{gathered}
$$

The brackets above mean integration over all angular variables.

This approach differs from the traditional expansions on $\mathrm{O}(3 N-3)$ harmonics due to the fact that the interactions are taken into account in the calculation of the angular functions. The obtainment of the potential curves is almost as difficult as the solution of the full problem; however, such decomposition has several advantages. The first of them is the physical interpretation of any quantum system in terms of potential curves and nonadiabatic couplings. A second important point is the energy independence of the potential curves. Once obtained, they can be used for both bound and continuum energy solutions. The potential curves are a universal characteristic of the system and do not depend on specific experimental situations. This means that excited states, resonances, and continuum properties in general can be studied by the same set of radial equations after the calculation of the angular solutions.

\section{ANGULAR SOLUTIONS FOR HELIUMLIKE ATOMS}

Our goal is to construct a new class of functions under some requirements. First of all, the set of functions should be able to solve the potential curve equation [6] for the threebody problems precisely, in full $R$-region with moderate computational efforts. Second, it should be useful for the general many-body problem as building blocks in the same sense that hydrogenic functions on Hartree solutions can be used for the many-electron atoms. This means that the func- 
tions should be generated by numerically exact computer codes and also that they should have analytical asymptotic and long-range properties.

\section{A. Angular equation}

Considering the nucleus (charge $Z$ ) as the center of mass, the hyperradius $R$ and the hyperangle $\alpha$ will be related to the spherical radial coordinates of the electrons $r_{1}$ and $r_{2}$ as given below:

$$
\begin{gathered}
r_{1}=R \sin \alpha, \quad r_{2}=R \cos \alpha, \\
R^{2}=r_{1}^{2}+r_{2}^{2}, \\
\tan \alpha=\frac{r_{1}}{r_{2}} .
\end{gathered}
$$

In atomic units, the angular hyperspherical equation for the channel functions and potential curves for this three-body problem is

$$
\begin{gathered}
{\left[\frac{d^{2}}{d \alpha^{2}}-\frac{\hat{l}_{1}^{2}}{\sin ^{2} \alpha}-\frac{\hat{l}_{2}^{2}}{\cos ^{2} \alpha}-\frac{2 Z R}{\sin \alpha}-\frac{2 Z R}{\cos \alpha}\right.} \\
\left.+\frac{2 R}{\sqrt{1-\sin (2 \alpha) \cos \theta}}-U_{\lambda}(R)\right] \\
\times(\sin \alpha \cos \alpha)^{-1} \Phi_{\lambda}(R ; \Omega)=0
\end{gathered}
$$

where $\hat{l}_{1}^{2}$ and $\hat{l}_{2}^{2}$ are the usual angular momentum operators and $\cos \theta=\hat{r}_{1} \cdot \hat{r}_{2}$. To preserve the individuality of the electrons with respect to the angular motion, the channel functions are now expanded in the basis of the coupled orbital angular momentum $\mathcal{Y}_{\ell_{1} \ell_{2}}^{L M}$ as

$$
\begin{aligned}
\Phi_{\lambda}(R ; \Omega)= & \sum_{\ell_{1} / 2}(\sin \alpha)^{\ell}{ }_{1}^{+1}(\cos \alpha)^{\ell}{ }_{2}+1 \\
& \times \mathcal{Y}_{\ell_{1} \ell_{2}}^{L M}\left(\hat{r}_{1}, \hat{r}_{2}\right) \mathcal{G}_{\ell_{1} \ell_{2}}^{\lambda}(R ; \alpha),
\end{aligned}
$$

where the total angular momentum $L$ is limited by the relation $\left|\ell_{1}-\ell_{2}\right| \leqslant L \leqslant \ell_{1}+\ell_{2}$ and the unit vectors $\hat{r}_{i}$ represent the angular spherical variables $\phi_{i}, \theta_{i}$ of the coordinate $\vec{r}_{i}$. The functions $\sin \alpha$ and $\cos \alpha$ take part of this expansion to eliminate the quadratic poles in the angular equation. The resulting equations are

$$
\begin{gathered}
{\left[\frac{d^{2}}{d \alpha^{2}}+2\left[\left(\ell_{1}+1\right) \cot \alpha-\left(\ell_{2}+1\right) \tan \alpha\right] \frac{d}{d \alpha}\right.} \\
\left.\quad-U_{\lambda}(R)-\left(\ell_{1}+\ell_{2}+2\right)^{2}\right] \mathcal{G}_{\ell_{1} \ell_{2}}^{\lambda}(R ; \alpha) \\
=R \sum_{\ell_{1}^{\prime} \ell_{2}^{\prime}} v_{\ell_{1} \ell_{2} \ell_{1}^{\prime} \ell_{2}^{\prime}}^{L M}(\alpha) \mathcal{G}_{\ell_{1}^{\prime} \ell_{2}^{\prime}}^{\lambda}(R ; \alpha),
\end{gathered}
$$

where

$$
\begin{aligned}
v_{\ell_{1} \ell_{2} \ell_{1}^{\prime} \ell_{2}^{\prime}}^{L M}(\alpha)= & (\sin \alpha)^{\ell_{1}^{\prime}-\ell_{1}}(\cos \alpha)^{\ell_{2}^{\prime}-\ell_{2}} \\
& \times\left\langle\mathcal{Y}_{\ell_{1} \ell_{2}}^{L M}|\hat{V}| \mathcal{Y}_{\ell_{1}^{\prime} \ell_{2}^{\prime}}^{L M}\right\rangle .
\end{aligned}
$$

At $R=0$, the interaction terms vanish and the function $\mathcal{G}^{\lambda} \ell_{1} \ell_{2}$ assumes the form

$$
\mathcal{G}^{\lambda} \ell_{1} \ell_{2}(0 ; \alpha)=P_{\mu}^{\ell} \ell_{1}+1 / 2, \ell_{2}+1 / 2(\cos 2 \alpha)
$$

where the functions $P_{\mu}^{/}{ }_{1}+1 / 2, \ell_{2}+1 / 2$ are the Jacobi polynomials [19]. The corresponding eigenvalues are

$$
U_{\lambda}(0)=-\left(2 \mu+\ell_{1}+\ell_{2}+2\right)^{2} \quad(\mu=0,1,2, \ldots)
$$

\section{B. Laguerre-Jacobi functions}

To make clear the topological properties of Eq. (14), we introduce the variable $z=\tan (\alpha / 2)$ [7]. This change provides coupled differential equations with rational coefficients that allows solutions by the use of Frobenius method. Unlike the expansion of the trigonometric coefficients of Eq. (14), previous studies [7] showed fast convergence of the expanded channel functions in power series in this new variable.

The $z$ variable is defined in the region $0 \leqslant z \leqslant 1$. For heliumlike atoms, the solutions can be limited to the region 0 $\leqslant z \leqslant(\sqrt{2}-1)$ by imposing Cauchy's continuity relations. Also, it is numerically convenient to impose polynomial solutions to the channel functions in the limits $R=0$ and $R$ $\rightarrow \infty$ by the following change:

$$
\mathcal{G}^{\lambda} \ell_{1} \ell_{2}(R ; z)=\left(1+z^{2}\right)^{-2 \mu} e^{p z} \mathcal{L}^{\lambda} \ell_{1} \ell_{2}(R ; z)
$$

where $p=-2 Z R / n_{\lambda}$, and $n_{\lambda}$ is the principal quantum number of the $\mathrm{He}^{+}$ion. This change produces the relations

$$
\begin{gathered}
{\left[\mathcal{A}(z) \frac{d^{2}}{d z^{2}}+\mathcal{B}_{\ell_{1} \ell_{2}}^{\mu}(z) \frac{d}{d z}+\mathcal{C}_{\ell_{1} \ell_{2}}^{\mu}(R ; z)\right] \mathcal{L}_{\ell_{1} \ell_{2}}^{\lambda}(R ; z)} \\
=R \sum_{\ell_{1}^{\prime} \ell_{2}^{\prime}} \mathcal{K}_{\ell_{1} \ell_{1}^{\prime} \ell_{2} \ell_{2}^{\prime}}(z) \mathcal{L}_{\ell_{1}^{\prime} \ell_{2}^{\prime}}^{\lambda}(R ; z) \\
\mathcal{A}(z)=z\left(1-z^{2}\right)\left(1+z^{2}\right)^{2} \\
\mathcal{B}_{\ell_{1} \ell_{2}}(z)=2\left(\ell_{1}+1\right)+2 p z-2\left(4 \mu+\ell_{1}+4 \ell_{2}+4\right) z^{2} \\
+2 p z^{3}-2\left(\ell_{1}+4 \ell_{2}+5\right) z^{4}-2 p z^{5} \\
+2\left(4 \mu+\ell_{1}\right) z^{6}-2 p z^{7}
\end{gathered}
$$




$$
\begin{aligned}
\mathcal{C}_{\ell_{1} \ell_{2}}^{\mu}(R ; z)= & 4 Z R+2 p\left(\ell_{1}+1\right)+\left[p^{2}+8 Z R-4 U_{\lambda}(R)\right. \\
& \left.-4\left(\ell_{1}+\ell_{2}+2\right)^{2}-4 \mu\left(2 \ell_{1}+3\right)\right] z \\
& -2 p\left(4 \mu+\ell_{1}+4 \ell_{2}+4\right) z^{2} \\
& +\left[p^{2}+8 Z R+4\left(\ell_{1}+\ell_{2}+2\right)^{2}+4 U_{\lambda}(R)\right. \\
& \left.+16 \mu\left(\ell_{1}+2 \ell_{2}+3\right)\right] z^{3} \\
& -2\left[2 Z R+\left(\ell_{1}+4 \ell_{2}+5\right) p\right] z^{4} \\
& -\left[p^{2}+16 \mu^{2}+4 \mu\left(2 \ell_{1}+1\right)\right] z^{5} \\
& +2 p\left(4 \mu+\ell_{1}\right) z^{6}-p^{2} z^{7}
\end{aligned}
$$

with the coupling term

$$
\begin{aligned}
\mathcal{K}_{\ell_{1} \ell_{2} \ell_{1}^{\prime} \ell_{2}^{\prime}}(z)= & \left(1+z^{2}\right) \sum_{J=J_{\min }}^{J_{\max }} 2^{\ell_{1}^{\prime}-\ell_{1}+3+J_{2} \ell_{1}^{\prime}-\ell_{1}+1+J} \\
& \times\left(1-z^{2}\right)^{\prime}-\ell_{2}-J \chi_{\ell_{1} \ell_{2} \ell_{1}^{\prime} \ell_{2}^{\prime}}^{L J}
\end{aligned}
$$

where $J_{\min }=\max \left(\left|\ell_{1}-\ell_{1}^{\prime}\right|,\left|\ell_{2}-\ell_{2}^{\prime}\right|\right)$ and $J_{\max }=\min \left(\ell_{1}\right.$ $\left.+\ell_{1}^{\prime}, \ell_{2}+\ell_{2}^{\prime}\right)$. The tensor $\chi_{\ell_{1} \ell_{2} \ell_{1}^{\prime} \ell_{2}^{\prime}}^{L}$ can be defined using the $3-j$ and $6-j$ notations as follows:

$$
\begin{aligned}
\chi_{\ell_{1} \ell_{2} \ell_{1}^{\prime} \ell_{2}^{\prime}=}^{L J} & (-1)^{J+L}\left[\left(2 \ell_{1}+1\right)\left(2 \ell_{2}+1\right)\right. \\
& \left.\times\left(2 \ell_{1}^{\prime}+1\right)\left(2 \ell_{2}^{\prime}+1\right)\right]^{1 / 2}\left(\begin{array}{ccc}
\ell_{1} & \ell_{1}^{\prime} & J \\
0 & 0 & 0
\end{array}\right) \\
& \times\left(\begin{array}{ccc}
\ell_{2} & \ell_{2}^{\prime} & J \\
0 & 0 & 0
\end{array}\right)\left\{\begin{array}{lll}
\ell_{1} & \ell_{2} & L \\
\ell_{2}^{\prime} & \ell_{1}^{\prime} & J
\end{array}\right\} .
\end{aligned}
$$

The Laguerre-Jacobi functions $\mathcal{F}^{\mu}{ }_{1} l_{2}(R ; z)$ are obtained as eigenstates of the decoupled terms of Eq. (19), or explicitly,

$$
\begin{aligned}
{[\mathcal{A}(z)} & \left.\frac{d^{2}}{d z^{2}}+\mathcal{B}_{\ell_{1} \ell_{2}}(z) \frac{d}{d z}+\mathcal{C}_{\ell_{1} \ell_{2}}(z)-R \mathcal{K}_{\ell_{1} \ell_{1} \ell_{2} \ell_{2}}(z)\right] \\
& \times \mathcal{F}_{\ell_{1} \ell_{2}}^{\mu}(R ; z) \\
= & u_{\ell_{1} \ell_{2}}^{\mu}(R) \mathcal{F}_{\ell_{1} \ell_{2}}^{\mu}(R ; z)
\end{aligned}
$$

The variation of the parameter $R$ from zero to infinity builds potential curves $u_{\ell_{1} \ell_{2}}^{\mu}(R)$ for the eigenstates $\mathcal{F}_{l_{1} \ell_{2}}^{\mu}(R ; z)$. The structure of this equation allows the use of the Frobenius method to obtain the function $\mathcal{F}_{l_{1} l_{2}}^{\mu}$, which is expanded in the form below:

$$
\mathcal{F}_{l_{1} \ell_{2}}^{\mu}(R ; z)=\sum_{k} A_{\ell_{1} \ell_{2}}^{\mu}(R ; k) z^{k}
$$

One of the main characteristics of this expansion is its polynomial form at the limits $R=0$ and $R \rightarrow \infty$. At $R=0$, the functions are

$$
\begin{aligned}
\mathcal{F}_{\ell_{1} \ell_{2}}^{\mu}(0 ; z)= & \sum_{m=0}^{\mu}\left(\begin{array}{c}
\mu+\ell_{1}+1 / 2 \\
m
\end{array}\right)\left(\begin{array}{c}
\mu+\ell_{2}+1 / 2 \\
\mu-m
\end{array}\right) \\
& \times(-1)^{\mu-m}(2 z)^{2(\mu-m)}\left(1-z^{2}\right)^{2 m},
\end{aligned}
$$

with the eigenvalues

$$
u_{\ell_{1} \ell_{2}}^{\mu}(0)=-\left(2 \mu+\ell_{1}+\ell_{2}+2\right)^{2} .
$$

At $R \rightarrow \infty$, the expressions for the eigenstates has the following Laguerre polynomial structure:

$$
\begin{aligned}
\mathcal{F}_{\ell_{1} \ell_{2}}^{\mu}(R \rightarrow \infty ; z) & =L_{n_{\lambda}-\ell_{1}-1}^{2 \ell_{1}+1}(\rho) \\
& =\sum_{m=0}^{n_{\lambda}-\ell_{1}-1}(-1)^{m}\left(\begin{array}{c}
n_{\lambda}+\ell_{1} \\
n_{\lambda}-\ell_{1}-1+m
\end{array}\right) \frac{\rho^{m}}{m !},
\end{aligned}
$$

where $\rho=2 Z R z$ and $L_{n_{\lambda}-\ell_{1}-1}^{2 \ell_{1}+1}(\rho)$ are the Laguerre polynomials [19]. In this limit, the potential curves have a defined asymptotic form:

$$
\begin{aligned}
u_{\ell_{1} \ell_{2}}^{\mu}(R)= & \frac{Z^{2}}{n_{\lambda}^{2}}+\frac{2(Z-1)}{R}+\frac{n_{\lambda}^{2}-2 \ell_{1}\left(\ell_{1}+1\right)-\ell_{2}\left(\ell_{2}+1\right)}{2 R^{2}} \\
& +O\left(\frac{1}{R^{3}}\right)
\end{aligned}
$$

where higher corrections could be obtained using perturbative methods [20]. The asymptotic quantum number that defines each potential curve at the dissociation limit is related to the set $\left\{\mu, \ell_{1}, \ell_{2}\right\}$ through the relation

$$
n_{\lambda}= \begin{cases}\frac{\mu}{2}+\ell_{1}+1 & \mu \text { even } \\ \frac{\mu}{2}+\ell_{1}+\frac{1}{2} & \mu \text { odd }\end{cases}
$$

which is no longer valid for eigenvalues of the coupled angular equation (14) as the nondiagonal terms force the "avoided crossings" of the potential curves.

\section{APPLICATION OF THE METHOD}

In this section, we analyze the performance of LaguerreJacobi functions on the diagonalization of the hyperspherical angular equation. The chosen system is the $\mathrm{Li}^{+}$ion with $L$ $=0$ and total $\operatorname{spin} S=0$. This is a good system to deal with as it can be compared with the well-known solutions and, using the $z$ variable, we can solve the coupled angular equation directly to compare the results.

Initially, the decoupled differential equations (25) are solved by Frobenius method for each value of the quantum numbers $\mu$ and $\ell$ up to the maximum numbers $\mu_{\max }$ and $\ell_{\text {max }}$, where $\ell=\ell_{1}=\ell_{2}$ for $L=0$. The resulting potential curves, labeled by the pair $(\mu, \ell)$, are shown in Figs. 1(a) and 1 (b) where we can see the lowest-energy spectrum. Con- 

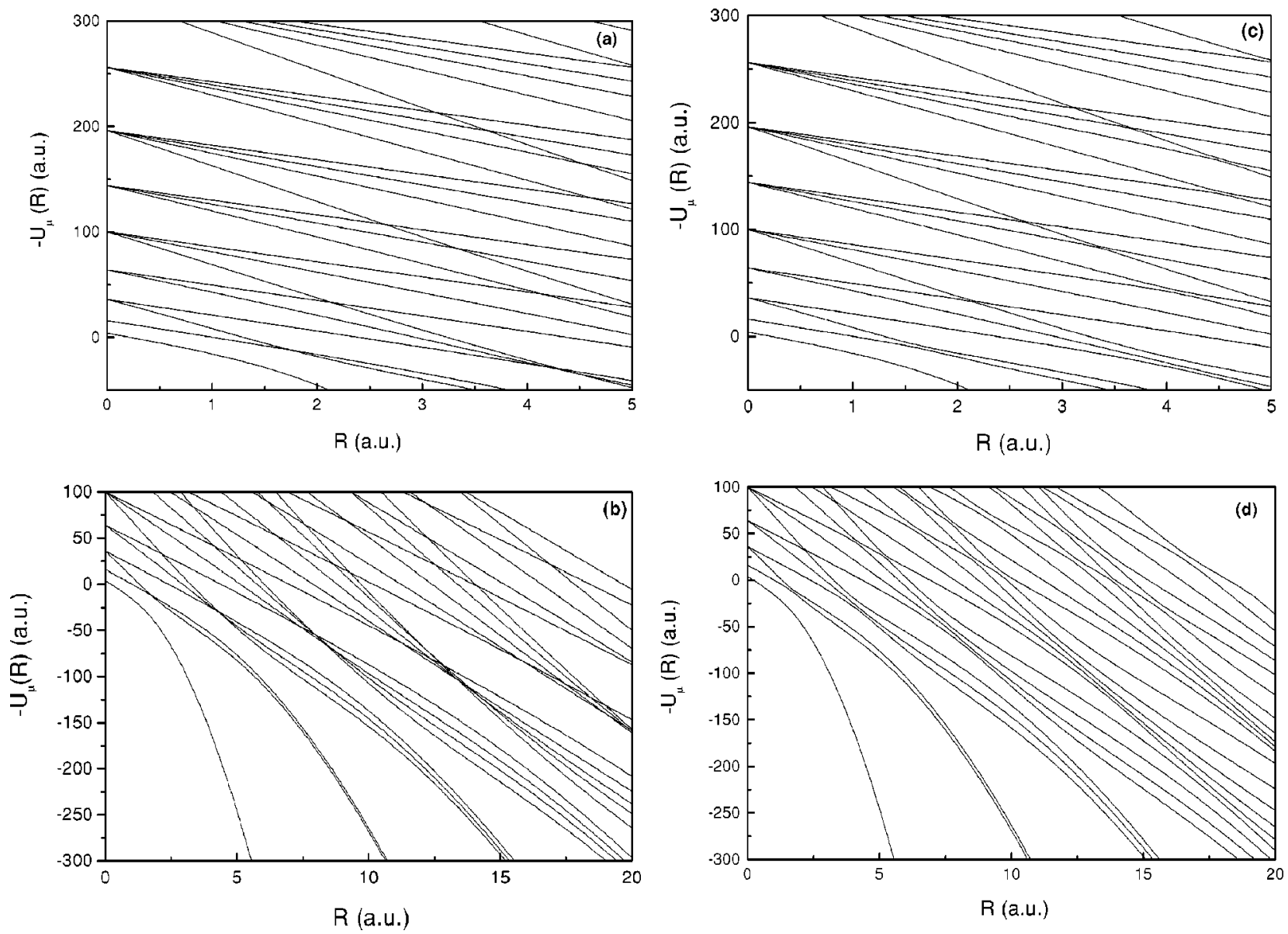

FIG. 1. Potential curves of the one-channel functions compared with the results of the coupled angular equation. (a) for the decoupled solution and (c) for the coupled one are shown close to the origin to show their similarity. The main difference between the decoupled and exact solution in (b) and (d), respectively, is related to the avoided crossing of the curves on the exact solution due to the coupling between angular components of different angular momenta.

sidering that different angular momentum solutions are not coupled by the interaction terms, the potential curves may cross each other. Besides, it is possible to associate the asymptotic quantum numbers with those numbers at $R=0$, as in Eq. (31). The eigenstates of these potential curves obtained from unidimensional equations are easily calculated with great precision for all of the range of the hyperradius.

After the normalization of the angular basis functions, we proceed to the diagonalization of the total angular operator. The resulting corrected potential curves are shown in Figs. 1(c) and 1(d) and the efficiency of the process can be verified in Table I, where we show the convergence of the lowest and most important potential curve for some representative values of $R$ with the varying size of the diagonalization matrix. The maximum value of the angular momentum $\ell_{\max }$ is related with the truncation of the expansion of the channel functions in the total angular momentum basis as given in Eq. (13). We can see that the convergence in $\mu_{\max }$ is very fast, for all values of $\ell_{\max }$ listed and for all regions of $R$. The angular momentum expansion is not as fast or efficient, especially for the minimum of the potential curve, but the choice of the coupled angular momentum is standard in the literature as it allows the simultaneous diagonalization of the angular operators of each electron.
We note that the curves in Figs. 1(a) and 1(b) resemble those of Figs. 1(c) and 1(d), except that the crossings are avoided in the latter. This means that Eq. (31) is no longer valid. In the region of the avoided crossings, the angular functions have sharp transitions as the behavior of the two angular channels changes into one another. This is reflected on the nonadiabatic couplings, especially on the $Q_{\lambda \lambda^{\prime}}$ 's, which involve second derivatives of the angular functions. An example is shown in Fig. 2 where some representative couplings between the first three potential curves present peaks related to the avoided crossings of the potential curves. The avoided crossings also affect the behavior of the angular channel functions generating sharp transitions, which are not present in the physical system. Their effect is further corrected by the radial coefficients $F_{\mu}(R)$ of the adiabatic expansion for each energy of the system.

The potential curves of the Laguerre-Jacobi solutions can be compared with the exact ones in a more familiar picture when divided by $R^{2}$, as shown in Fig. 3. We observe that, except in the avoided-crossing regions, the deviations are more pronounced on the minimum of the potential well as expected, since the interaction potential for small and asymptotic values of $R$ is not strongly dependent on the offdiagonal angular momentum coupling. 
TABLE I. Convergence of the diagonalizing process for the first potential curve. The indices $\mu_{\max }$ and $\ell_{\max }$ are the maximum value of the Jacobi and angular momentum labels used to construct the diagonalization basis. The resulting matrix has the dimension $\ell_{\max } \mu_{\max } / 2$ ( $\mu$ is even for $L=0, S=0$ states). The point $R=0.45$ a.u. corresponds approximately to the minimum of the potential curve well.

\begin{tabular}{|c|c|c|c|c|c|c|}
\hline$R$ (a.u.) & $\ell_{\max }$ & 2 & 4 & 6 & 8 & 9 \\
\hline \multirow[t]{6}{*}{0.04} & $\mu_{\max }$ & & & & & \\
\hline & 2 & -3.6401197175 & -3.640119713 & -3.6401197122 & -3.640119712 & -3.640119712 \\
\hline & 6 & -3.6401142350 & -3.640114223 & -3.6401142214 & -3.640114221 & -3.640114221 \\
\hline & 10 & -3.6401136032 & -3.640113587 & -3.6401135847 & -3.640113584 & -3.640113584 \\
\hline & 14 & -3.6401134455 & -3.640113427 & -3.6401134239 & -3.640113423 & -3.640113423 \\
\hline & 18 & -3.6401133894 & -3.640113369 & -3.6401133660 & -3.640113365 & -3.640113365 \\
\hline \multicolumn{7}{|l|}{0.45} \\
\hline & 2 & 4.3647270127 & 4.364729119 & 4.364729321 & 4.364729358 & 4.364729365 \\
\hline & 6 & 4.3667106292 & 4.366715245 & 4.366715771 & 4.366715880 & 4.366715901 \\
\hline & 10 & 4.3669268933 & 4.366932873 & 4.366933634 & 4.366933806 & 4.366933839 \\
\hline & 14 & 4.3669799868 & 4.366986747 & 4.366987673 & 4.366987894 & 4.366987938 \\
\hline & 18 & 4.3669987368 & 4.367005967 & 4.367007010 & 4.367007269 & 4.367007322 \\
\hline \multicolumn{7}{|l|}{2.00} \\
\hline & 2 & 45.400931036 & 45.40093327 & 45.40093357 & 45.40093363 & 45.40093364 \\
\hline & 6 & 45.404243197 & 45.40424797 & 45.40424865 & 45.40424881 & 45.40424884 \\
\hline & 10 & 45.404533682 & 45.40453976 & 45.40454068 & 45.40454090 & 45.40454094 \\
\hline & 14 & 45.404599778 & 45.40460658 & 45.40460766 & 45.40460793 & 45.40460798 \\
\hline & 18 & 45.404622325 & 45.40462957 & 45.40463075 & 45.40463106 & 45.40463112 \\
\hline \multicolumn{7}{|l|}{10.00} \\
\hline & 2 & 940.56783259 & 940.5679039 & 940.5679334 & 940.5679428 & 940.5679446 \\
\hline & 6 & 940.56783355 & 940.5679056 & 940.5679354 & 940.5679448 & 940.5679466 \\
\hline & 10 & 940.56783355 & 940.5679056 & 940.5679354 & 940.5679448 & 940.5679466 \\
\hline & 14 & 940.56783355 & 940.5679056 & 940.5679354 & 940.5679448 & 940.5679466 \\
\hline & 18 & 940.56783355 & 940.5679056 & 940.5679354 & 940.5679448 & 940.5679466 \\
\hline
\end{tabular}

In order to analyze the effect of the size of the diagonalization basis in the angular channel functions as a function of $\alpha$, we have squared the channel function and integrated it over the spherical angles $\Omega_{i}=\left\{\theta_{i}, \phi_{i}\right\}, i=1,2$. Such a procedure is defined as follows:

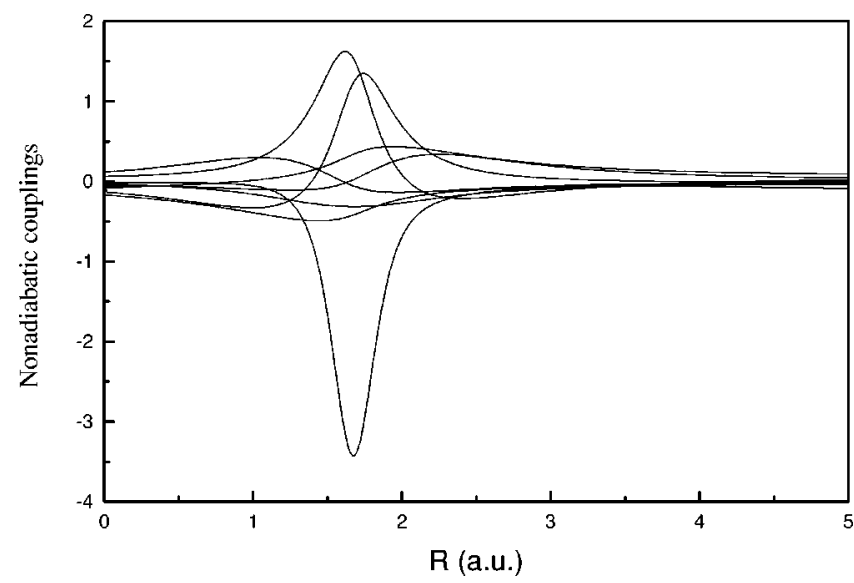

FIG. 2. Some representative nonadiabatic couplings (in atomic units) for the first three angular channels. The largest peaks are related to inflections of the potential curves, becoming especially sharp in the region of avoided-crossings.

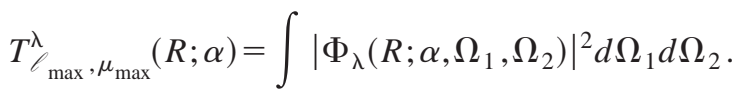

Using the channel function corresponding to the lowest potential curve $(\lambda=1)$, we have computed the functional (32), for different values of $R$, using only one Laguerre-Jacobi function, labeled by the pair $\left(\ell_{\max }, \mu_{\max }\right)=(0,0)$, and then using $\ell_{\max }=9$ and $\mu_{\max }=9$. The comparison between these two calculations is shown in Fig. 4. In this figure we can see that there is no apparent difference between both calculations for each chosen value of $R$. This difference is shown in detail in Fig. 5, where it can be observed that even for the minimum of the potential curve, about $R=0.45$ a.u., the difference between the solutions is very small. This is a strong indication of the appropriated choice of the diagonalization basis. It is important to note that the good result is due to the continuous transformation of the angular basis as the hyperradius changes, avoiding the necessity of different approaches for each region of $R$. An example of such a case would be the analytical solutions at $R=0$, which fail to reproduce the asymptotic behavior, and also the hydrogenic solutions for large values of $R$, which lack completeness unless the hard-dealing continuum solutions are taken into account. 

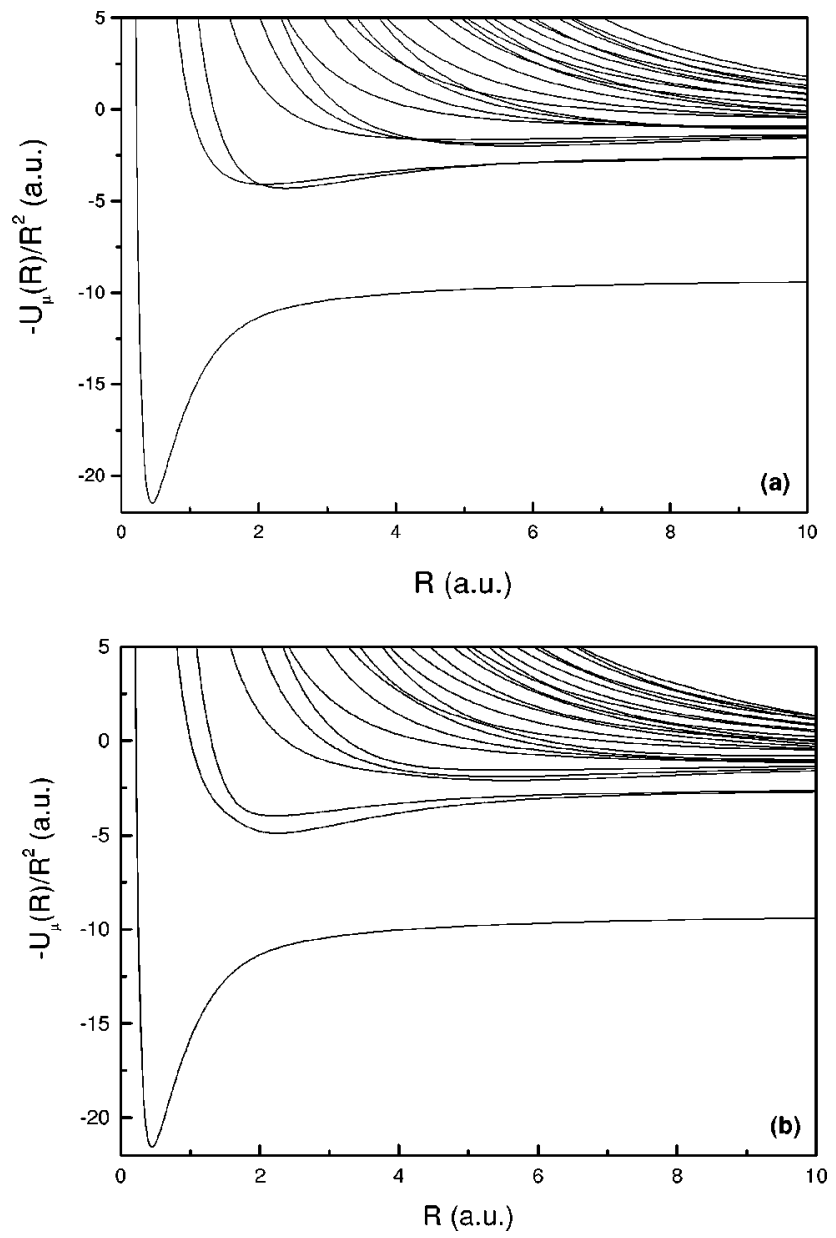

FIG. 3. Comparison between potential curve wells of the $\mathrm{Li}^{+}$. (a) Decoupled solutions. (b) The exact solutions. The lowest potential curve, which gives the bound states, is deeper for the decoupled solution than for the exact one since it does not take into account all the effects of the electron-electron repulsion. For large and small $R$, the differences between the solutions become smaller.

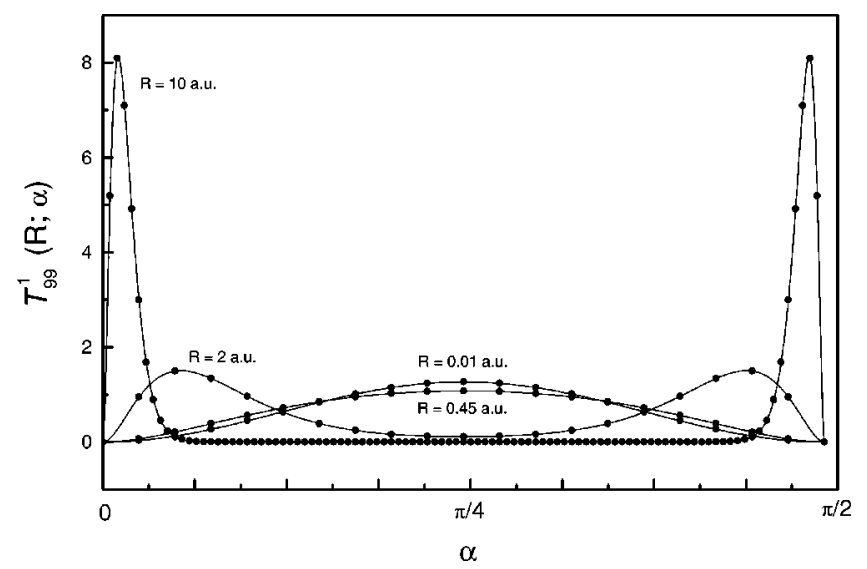

FIG. 4. Behavior of the coupled angular channel functions (in atomic units) as given by Eq. (32), with $\left(\mu_{\max }, \ell_{\max }\right)=(9,9)$ for different values of the hyperradius. The dots are the same calculation using only the lowest basis eigenstates, which corresponds to $\left(\mu_{\max }, \ell_{\max }\right)=(0,0)$.
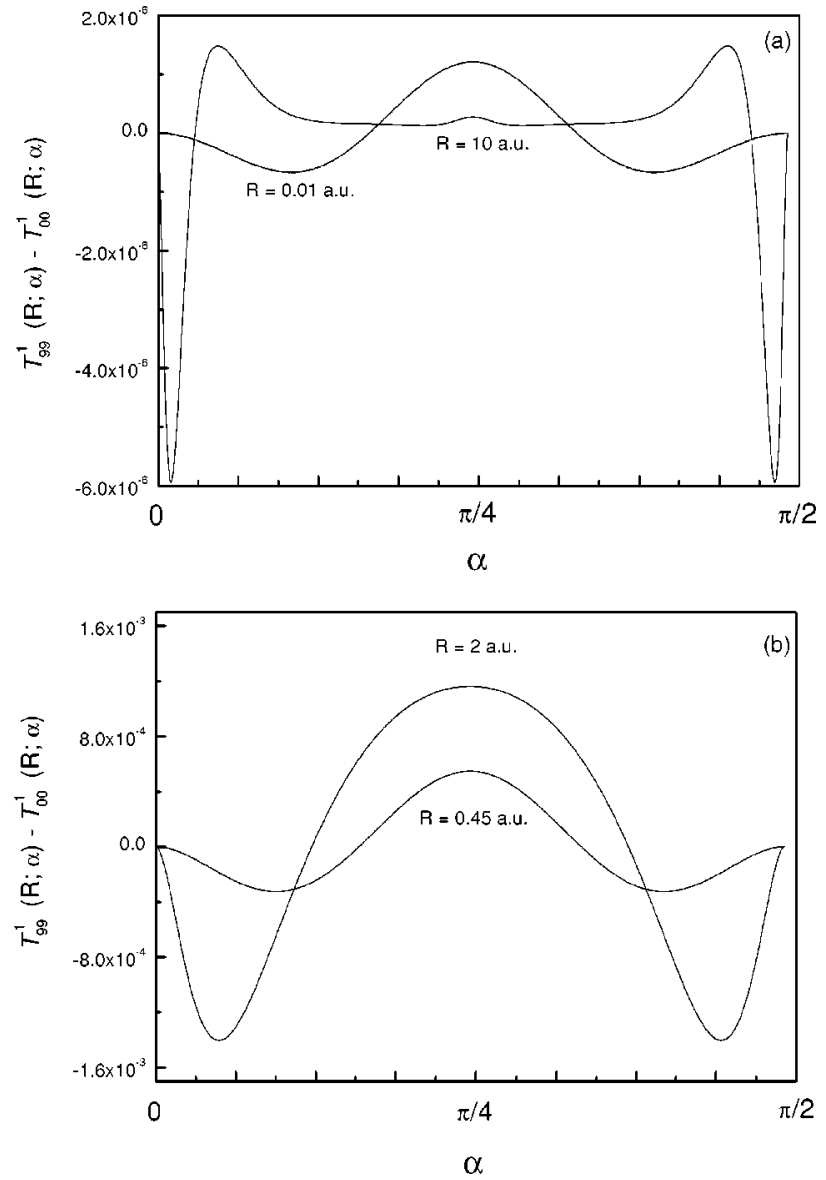

FIG. 5. Difference between the solutions shown in Fig. 4. (a) For small and large values of $R$. (b) For intermediate values of $R$.

With the angular solutions calculated, we can now solve the radial equation for the bound states of the $\mathrm{Li}^{+}$. The determination of the energy lines is done in a systematic way by truncating the adiabatic expansion into a maximum number $N_{c}$ of coupled channels. The calculated energy for each approximation is related to the exact value in an upper and lower bound scheme $[21,22]$, i.e.,

$$
\varepsilon_{\mathrm{EAA}} \leqslant \varepsilon_{\text {exact }} \leqslant \varepsilon_{\mathrm{CAA}} \leqslant \varepsilon_{\mathrm{UAA}},
$$

where the EAA (extreme adiabatic approximation) approach corresponds to neglecting all couplings, the UAA (uncoupled adiabatic approximation) corresponds to the inclusion of the diagonal coupling, and the nondiagonal couplings are taken into account on the CAA (coupled adiabatic approximation), approaching the exact energy as more radial channels are coupled. This behavior is clear in Table II, where the calculated energy converges to the variational result [23] as the number of $N_{c}$ coupled channels increases. The convergence is not uniform because channels related with the same angular momentum of the first channel are expected to give the most important contributions. With 13 coupled channels, the error obtained is less than $1 \mathrm{ppm}$. In the same table, we show that the convergence pattern is similar to the helium case [18], even with the significative difference in the calculated energy. This suggests the use of this basis for the calculation of energies in the isoelectronic series of the helium. 
TABLE II. Ground-state energy $\left[\varepsilon_{0}\right.$ (a.u.)] convergence of the $\mathrm{Li}^{+}$as a function of the number $N_{c}$ of coupled angular states in the radial equations. It used 30 coupled angular momenta for the first potential curve and for the corresponding diagonal nonadiabatic coupling $Q_{11}$. All other potential curves were obtained using 10 coupled angular momenta. The first row, $1^{*}$, corresponds to the lower bound calculation (EAA approach). The variational result $\left(\varepsilon_{\mathrm{var}}\right)$ is from Ref. [23]. The convergence follows a similar pattern to that observed for the He atom [18].

\begin{tabular}{cccr}
\hline \hline$N_{c}$ & Energy $\left(-\varepsilon_{0}\right)$ & $\left(\varepsilon_{\mathrm{var}}-\varepsilon_{0}\right) / \varepsilon_{\mathrm{var}}(\mathrm{ppm})$ & \multicolumn{1}{c}{ He $[18]$} \\
\hline $1^{*}$ & 7.33234538 & -7202.28 & -9060.02 \\
1 & 7.26264006 & 2372.74 & 2813.91 \\
2 & 7.26701680 & 1771.53 & 1748.85 \\
3 & 7.27970490 & 28.64 & 38.88 \\
4 & 7.27973128 & 25.02 & 31.66 \\
5 & 7.27973427 & 24.60 & 30.30 \\
6 & 7.27975647 & 21.55 & 22.69 \\
7 & 7.27989774 & 2.15 & 2.52 \\
8 & 7.27989778 & 2.14 & 2.50 \\
9 & 7.27989790 & 2.13 & 2.46 \\
10 & 7.27989792 & 2.13 & 2.42 \\
11 & 7.27989797 & 2.12 & \\
12 & 7.27989842 & 2.06 & \\
13 & 7.27990925 & 0.57 & \\
14 & 7.27990926 & 0.57 & \\
15 & 7.27990927 & 0.57 & \\
16 & 7.27990928 & 0.56 & \\
17 & 7.27990928 & 0.56 & \\
18 & 7.27990929 & 0.56 & \\
19 & 7.27990929 & 0.56 & \\
20 & 7.27990931 & 0.56 & \\
21 & 7.27991083 & 0.35 & \\
\hline \hline
\end{tabular}

The first excited-state energies are listed in Table III. They are obtained from the same set of potential curves as the ground-state energy, but there is a loss of accuracy for the lowest states due to the behavior of nonadiabatic couplings with $R$. This effect becomes less important for higher excited states, whose wave functions' main bodies are distributed over larger values of $R$, where the couplings are very small, as seen in Fig. 2.

\section{CONCLUSION}

The solution of a system of partial equations in $3 N-4$ angular variables is a formidable and sometimes unpractical task. Direct solutions are difficult even in the $N=3$ heliumlike case. The long-range interactions cause three different regimes, in which the solutions differ totally. Aside from the spherical harmonics, the $R=0$ free particle functions are Jacobi polynomials in essence. In the asymptotic region, the bound behavior will dominate (hydrogenic in the helium case) and the intermediate region is a transition between both behaviors. Therefore, a proper numerical technique for one region will be inaccurate and instable in the other regions. These problems are significative due to the fact that channel functions and potential curves are the basic input for the
TABLE III. Lowest binding energies, $\varepsilon$ (a.u.), of the $\mathrm{Li}^{+}$for $N_{c}=21$ coupled angular states in the radial equations compared with the variational energies $\varepsilon_{\text {var }}$ from Ref. [24], except for the ground-state energy, which is from Ref. [23].

\begin{tabular}{cccc}
\hline \hline State & $\begin{array}{c}\text { Energy } \\
(-\varepsilon)\end{array}$ & $\begin{array}{c}\text { Variational }\left(-\varepsilon_{\mathrm{var}}\right) \\
{[24]}\end{array}$ & $\begin{array}{c}\left(\varepsilon_{\mathrm{var}}-\varepsilon\right) / \varepsilon_{\mathrm{var}} \\
(\mathrm{ppm})\end{array}$ \\
\hline $1 \mathrm{~s} 1 \mathrm{~s}$ & 7.2799108 & 7.27991339 & 0.35 \\
$1 \mathrm{~s} 2 \mathrm{~s}$ & 5.0408659 & 5.04087674 & 2.15 \\
$1 \mathrm{~s} 3 \mathrm{~s}$ & 4.7337250 & 4.733756 & 6.55 \\
$1 \mathrm{~s} 4 \mathrm{~s}$ & 4.6297491 & 4.629783 & 7.34 \\
$1 \mathrm{~s} 5 \mathrm{~s}$ & 4.5824015 & 4.582427 & 5.57 \\
\hline \hline
\end{tabular}

radial equations. Inaccuracies on those quantities will deteriorate the calculation of energies and radial amplitudes.

The method of analytical expansions developed in Ref. [7] solves the problems for most of the three-body systems. The understanding that the expansion in harmonics associated to the $\mathrm{O}(3 N-3)$ symmetry is not efficient even in the intermediate $R$-region suggested the solution of the equations by power series in an appropriated angular variable. The extension of the method for more complex problems is, however, unpractical. The $N>3$ problem requires several angular variables and therefore multivariable power expansion techniques are extremely difficult. However, the calculation of potential curves for $N>3$ can proceed by the construction of a new class of one-dimensional $R$-dependent functions to replace the ordinary Jacobi basis.

The eigenstates of the decoupled angular equation, the one-channel functions, fill those requirements when the variable $z$ is introduced. With this variable, the angular differential equation for each channel may be changed to furnish polynomial solutions at the limits $R=0$ and $R \rightarrow \infty$. The use of the Frobenius method leads to very fast convergent expansions of the eigenstates in the full $R$ region. The main aspect of the angular basis constructed with these functions is the update of the basis with $R$ as it carries the information of the diagonal components of the interaction. The result of this procedure is the fast convergence in all of the diagonalization process, especially at the dissociation region. For the positive ion of the lithium, the energy obtained with these potential curves has an accuracy of a few parts per million.

In this paper, we show a significant gain in efficiency diagonalizing the angular heliumlike atom equation with the one-channel basis instead of the pure hyperspherical harmonics. The hope to use it for the many-body problem functions generated by the three-body problems lies in the fact that the kinetic-energy operator in hyperspherical coordinates is constructed recursively from lower dimensions to higher ones.

\section{ACKNOWLEDGMENTS}

This work was supported by the Brazilian Agencies Conselho Nacional de Desenvolvimento Científico e Tecnológico $(\mathrm{CNPq})$ and Fundação de Amparo à Pesquisa do Estado de São Paulo (FAPESP), Processes Nos. 98/03044-7 and 97/06271-1. 
[1] C.D. Lin and X. Liu, Phys. Rev. A 37, 2749 (1988).

[2] J.J. De Groote, J.E. Hornos, H.T. Coelho, and C.D. Caldwell, Phys. Rev. B 46, 2101 (1992).

[3] O.I. Tolstikhin, S. Watanabe, and M. Matsuzawa, Phys. Rev. Lett. 74, 3573 (1995).

[4] J. Brust and C.H. Greene, Phys. Rev. A 56, 2005 (1997).

[5] S. Schmatz and D.C. Clary, J. Chem. Phys. 110, 9483 (1999).

[6] J.H. Macek, J. Phys. B 1, 831 (1968).

[7] J.E. Hornos, S.W. MacDowell, and C.D. Caldwell, Phys. Rev. A 33, 2212 (1986).

[8] A.G. Abrashkevich, D.G. Abrashkevich, M.I. Gaysak, V.I. Lendyel, I.V. Puzynin, and S.I. Vinitsky, Phys. Lett. A 152, 467 (1991).

[9] J.Z. Tang, S. Watanabe, and M. Matsuzawa, Phys. Rev. A 46, 2437 (1992).

[10] C.D. Lin, Phys. Rep. 257, 1-83 (1995).

[11] C.D. Lin, Phys. Rev. A 57, 4268 (1998).

[12] Yu.F. Smirnov and K.V. Shitikova, Fiz. Elem. Chastits At. Yadra 8847 (1977) [Sov. J. Part. Nucl. 8, 344 (1977)].

[13] E. Santopinto, F. Iachello, and M.M. Giannini, Eur. Phys. J. A
1, 307 (1998).

[14] L.V. Grigorenko, B.V. Danilin, V.D. Efros, N.B. Shul'gina, and M.V. Zhukov, Phys. Rev. C 60, 44312 (1999).

[15] H.T. Coelho, J.J. De Groote, and J.E. Hornos, Phys. Rev. A 46, 5443 (1992).

[16] J.J. De Groote, A.S. dos Santos, M. Masili, and J.E. Hornos, Phys. Rev. B 58, 10383 (1998).

[17] M. Masili, J.J. De Groote, and J.E. Hornos, Phys. Rev. A 52, 3362 (1995).

[18] J.J. De Groote, M. Masili, and J.E. Hornos, J. Phys. B 31, 4755 (1998).

[19] Handbook of Mathematical Functions, 3rd ed. edited by M. Abramowitz and I. A. Stegun (Dover, New York, 1965).

[20] J. Macek, Phys. Rev. A 31, 2162 (1985).

[21] H.T. Coelho and J.E. Hornos, Phys. Rev. A 43, 6379 (1991).

[22] A.F. Starace and G.L. Webster, Phys. Rev. A 19, 1629 (1979).

[23] G.W. Drake, Can. J. Phys. 66, 586 (1988).

[24] Y. Accad, C.L. Pekeris, and B. Schiff, Phys. Rev. A 4, 516 (1970). 